\title{
Failure to Reduce: Small Bowel Obstruction Hidden Within a Chronic Umbilical Hernia Sac
}

Caitlin P. Schrepel, MD; Fiona Gallahue, MD

\section{A 45-year-old woman with a history of an umbilical hernia presented with 5 hours of pain at the site of her hernia.}

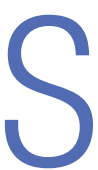

trangulated hernias are a medical emergency that can lead to small bowel obstruction (SBO), bowel necrosis, and death. Practitioners look for signs of strangulation on examination to guide the urgency of management. If the hernia is soft and reducible without overlying skin changes or signs of obstruction, patients may be monitored for years. ${ }^{1}$ However, there is increasing evidence that even asymptomatic hernias should be repaired rather than monitored to avoid the need for emergent surgical intervention. ${ }^{1}$

We present a case of a patient with a chronic umbilical hernia who experienced acute worsening of pain at the site of her hernia but with few additional objective signs of strangulation. Prior to this presentation, she had been recently evaluated at our ED for the "same" pain, which included a computed tomography (CT) scan that was negative for an acute surgical emergency. The patient's second ED visit led to a diagnostic dilemma: Practitioners are encouraged to avoid "unnecessary" radiation-especially in cases of chronic pain-and to rely on history, physical examination findings, and prior recent imaging studies, as appropriate. In this case, repeat imaging ultimately revealed a surgical emergency with an unusual underlying pathology likely related to the chronicity of the patient's hernia, and explained her repeat presentation to the ED.

\section{Case}

A 45-year-old obese woman (body mass index, $46 \mathrm{~kg} / \mathrm{m}^{2}$ ) with a medical history of an umbilical hernia, tubal ligation, and chronic pelvic pain presented a second time to our ED with pain at the site of her hernia, which she stated began 5 hours prior to presentation. Although the pain was associated with nausea and vomiting, the patient said her bowel movements were normal. She first noticed the hernia more than 5 years ago, but experienced her first episode of acute pain related to the hernia with associated nausea and vomiting 3 weeks earlier, which prompted her initial presentation. During this first ED visit, a CT scan of the abdomen/pelvis was obtained as part of her evaluation and was significant for umbilical herniation of bowel without evidence of strangulation. Bedside reduction was successful, and the patient was discharged home and informed of the need to follow-up with a surgeon for an elective repair. She returned to the ED prior to her

Dr Schrepel is a second-year resident in the Division of Emergency Medicine, University of Washington, Seattle. Dr Gallahue is the residency program director for the Division of Emergency Medicine, University of Washington, Seattle.

Authors' Disclosure Statement: The authors report no actual or potential conflict of interest in relation to this article.

DOI: 10.12788/emed.2016.0067 


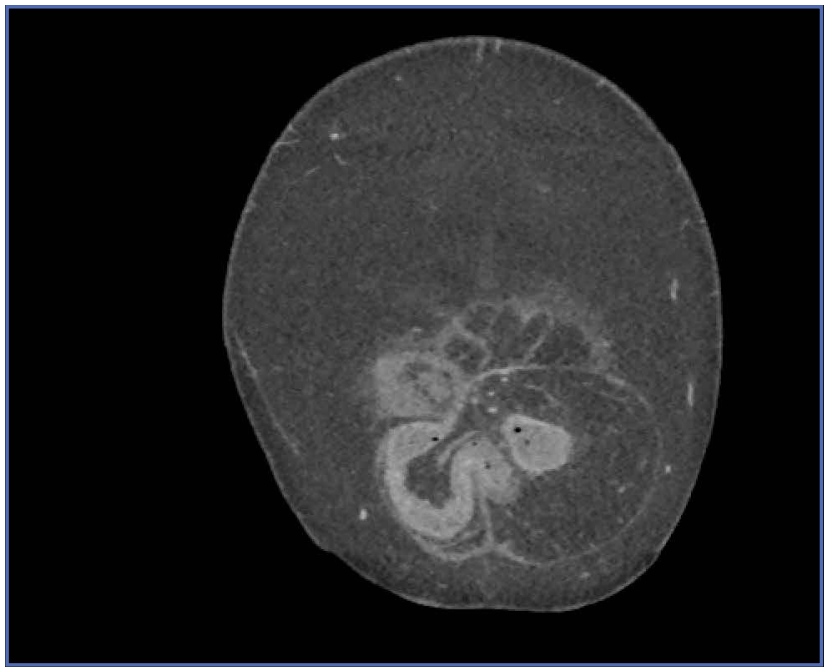

Figure 1. Computed tomography scan demonstrating a band-like structure at the six o'clock position, which likely represents an adhesion at the transition point of the small bowel obstruction.

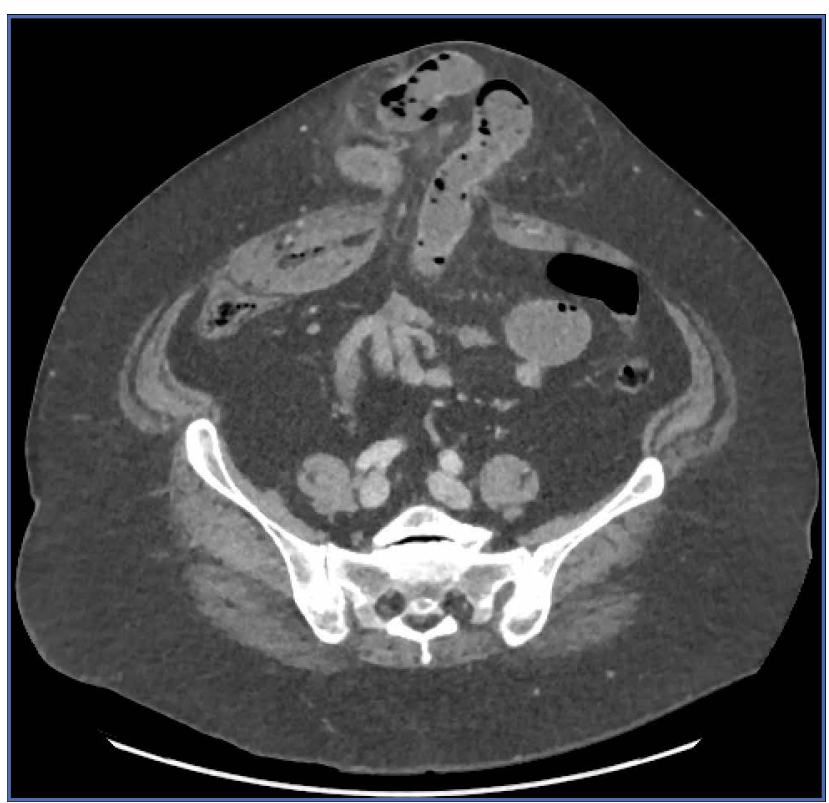

Figure 2. Computed tomography scan demonstrating an umbilical hernia with open umbilical hernia neck.

scheduled operation due to recurrent pain of similar character, but increased severity.

On physical examination, the patient was hemodynamically stable and afebrile. Her vital signs were: heart rate, 84 beats/ min; blood pressure, 113/68 mm Hg; and respiratory rate, 20 breaths/min. Oxygen saturation was $100 \%$ on room air.
The abdomen was soft with tenderness to palpation over a $13-\mathrm{cm} \times 8$-cm soft hernia to the left of the umbilicus without overlying skin changes. The patient's pain was controlled with $1 \mathrm{mg}$ of intravenous hydromorphone, after which bedside reduction was attempted. During reduction attempts, there was palpable bowel within the hernia sac, and a periumbilical defect was appreciated. Although the defect in the abdominal wall was estimated to be large enough to allow reduction, the hernia reduced only partially. Because imaging studies from the patient's previous ED visit showed no visualized strangulation or obstruction, we deliberated over the need for a repeat CT scan prior to further attempts at reduction by general surgery services. Ultimately, we ordered a repeat CT scan, which was significant for a "mechanical small bowel obstruction with focal transition zone located within the hernia sac itself, not the neck of the umbilical hernia." A band-like structure, likely representing an adhesion, was seen at the transition point (Figures 1 and 2). This adhesion was not visualized on the earlier CT study.

Small bowel obstruction is commonly caused by strangulation at the neck of a hernia. In this case, however, the patient had developed an adhesion within the hernia sac itself, which caused the obstruction. This explains why none of the overlying skin changes commonly found in strangulation were visible, and why we were unable to reduce the bowel even though we could palpate the large abdominal wall defect.

Following evaluation by general surgery services, the patient was admitted for laparoscopic hernia repair. Her case was transitioned to an open repair due to extensive intra-abdominal adhesions. The hernia was closed with mesh, and the patient recovered appropriately postoperatively.

\section{Discussion}

Abdominal wall hernias are a common pathology, with more than 700,000 repairs 
performed every year in the United States. ${ }^{2}$ Patients most commonly present to the ED with abdominal pain, nausea, and vomiting. Less frequently, they present with obstruction, incarceration, strangulation, or rupture. ${ }^{3}$ Umbilical hernias are caused by increasing intra-abdominal pressure. As the incidence of obesity in the United States has continued to increase, the proportion of hernias that are umbilical or periumbilical has also increased. ${ }^{2,4}$ Unfortunately, even though umbilical hernias are becoming more common, they are often given less attention than other types of hernias. ${ }^{5}$ The practice of solely monitoring umbilical hernias can lead to serious outcomes. For example, in a case presentation from Spain, a morbidly obese patient died due to a strangulated umbilical hernia that had progressed over a 15-year period without treatment. ${ }^{6}$

Compared to elective surgery, emergent operative repair is associated with a higher rate of postoperative complications, ${ }^{1}$ and a growing body of evidence suggests that patients with symptomatic hernias should be encouraged to undergo operative repair. ${ }^{1,6}$ However, the timing and urgency of this repair is often difficult to determine when strangulation is not the obvious diagnosis. Recent literature has shown that, even in nonincarcerated hernias, conservative management is a significant contributing factor in the delay of treatment, ultimately leading to complications, including SBO. ${ }^{1}$ Our patient had her umbilical hernia for over 5 years, during which time she had a tubal ligation and chronic pelvic pain, both of which could have led to intra-abdominal inflammation and the formation of adhesions. In her case, these adhesions led to an SBO, necessitating urgent surgery. This case supports the need to strongly consider ordering imaging studies to evaluate hernias when they cannot easily be reduced to avoid potential complications from attempting to reduce obstructed bowel, and to help determine surgical urgency, even when examination is otherwise benign. Additionally, while our patient had a good clinical outcome, the urgency of her procedure may further support surgical repair over conservative management of asymptomatic hernias.

\section{Conclusion}

Umbilical hernias have become more common with increasing rates of obesity. These hernias have the potential to lead to serious medical emergencies, and the common practice of monitoring chronic hernias may increase the patient's risk of serious complications. Emergency physicians use the physical examination to help determine the urgency of repair; however, imaging should be considered to assess hernias that cannot easily be reduced to evaluate for obstructed, strangulated, or incarcerated bowel and to help determine the urgency of surgical repair.

\section{References}

1. Davies M, Davies C, Morris-Stiff G, Shute K. Emergency presentation of abdominal hernias: outcome and reasons for delay in treatment-a prospective study. Ann R Coll Surg Engl. 2007;89(1):47-50.

2. Dabbas N, Adams K, Pearson K, Royle G. Frequency of abdominal wall hernias: is classical teaching out of date? JRSM Short Rep. 2011;2(1):5. doi:10.1258/ shorts.2010.010071.

3. Rodriguez JA, Hinder RA. Surgical management of umbilical hernia. Operat Tech Gen Surg. 2004;6(3): 156-164.

4. Aslani N, Brown CJ. Does mesh offer an advantage over tissue in the open repair of umbilical hernias? A systematic review and meta-analysis. Hernia. 2010;14(5):455-462. doi:10.1016/j.amjsurg.2011.11.015.

5. Arroyo A, García P, Pérez F, Andreu J, Candela F, Calpena R. Randomized clinical trial comparing suture and mesh repair of umbilical hernia in adults. Br J Surgery. 2001;8(10):1321-1323.

6. Rodríguez-Hermosa JI, Codina-Cazador A, Ruiz-Feliú B, Roig-García J, Albiol-Quer M, Planellas-Giné P. Incarcerated umbilical hernia in a super-super-obese patient. Obes Surg. 2008;18(7):893-895. doi:10.1007/ s11695-007-9397-3. 